http://dx.doi.org/10.1590/1678-6930

Arq. Bras. Med. Vet. Zootec., v.67, n.2, p.372-380, 2015

\title{
Avaliação da analgesia pós-operatória da metadona, da cetamina ou da sua associação em gatas submetidas a ovariossalpingo-histerectomia
}

[Evaluation of postoperative analgesia of methadone, ketamine or their associations in cats undergoing ovariohysterectomy]

\author{
V.S. Padilha ${ }^{1}$, R. Tocheto ${ }^{1}$, S. L. Beier ${ }^{2}$, J. Volpato ${ }^{1}$, N. Oleskovicz ${ }^{1}$
}

${ }^{1}$ Centro de Ciências Agroveterinárias - Universidade do Estado de Santa Catarina - CAV-UDESC - Lages, SC

${ }^{2}$ Escola de Veterinária - Universidade Federal de Minas Gerais - Belo Horizonte, MG

\section{RESUMO}

A prevenção e o controle da dor são componentes básicos para um procedimento anestésico adequado. Objetivou-se com este estudo avaliar a analgesia pós-operatória da cetamina, em dose subanestésica, da metadona e da associação de ambas pela via intramuscular em felinos. Foram utilizadas 24 gatas, hígidas, submetidas à ovariossalpingo-histerectomia eletiva. No dia anterior ao início do estudo, os animais foram anestesiados para colocação de cateter na veia jugular, para posterior coleta de sangue para mensuração da concentração sérica do cortisol. No dia do experimento, os animais foram alocados aleatoriamente em três grupos ( $\mathrm{n}=$ oito), os quais receberam cetamina (GC), na dose de $0,5 \mathrm{mg} / \mathrm{kg}$; ou metadona (GM), na dose de $0,3 \mathrm{mg} / \mathrm{kg}$; ou cetamina e metadona (GCM), nas doses de $0,5 \mathrm{mg} / \mathrm{kg}$ e $0,3 \mathrm{mg} / \mathrm{kg}$, respectivamente, todos pela via intramuscular. Todos os grupos receberam os fármacos 20 minutos antes da indução anestésica, a qual foi realizada com propofol, e a manutenção com isoflurano. Os parâmetros avaliados foram a mensuração do cortisol nos momentos M0 (basal - antes da cirurgia), M1(transoperatório), M2, M3, M4, M8, M12 e M24 (duas, três, quatro, oito, 12 e 24 horas após o término da cirurgia), e a do escore de dor por meio da escala multidimensional de dor aguda em felinos nos momentos M0, M2, M3, M4, M8, M12 e M24. Os valores de cortisol foram maiores no GC em comparação ao GM e ao GCM em M2 e M3. Os escores de dor foram maiores no GC nos momentos M2 e M3 em comparação ao GM e ao GCM, respectivamente. No somatório de pontos, no M2, o GC apresentou valores maiores que o GM e o GCM, e, no M4, o GC obteve valores maiores que o GCM. Em relação ao número de resgates no pósoperatório no GC, 8/8 dos animais necessitaram de resgate, no GM 5/8 e no GCM 3/8. Conclui-se que a associação de metadona e cetamina em gatas submetidas à OSH eletiva promove analgesia pós-operatória adequada e, assim, reduz o requerimento de analgésicos no período pós-operatório.

Palavras-chave: gato, cetamina, metadona, analgesia

\begin{abstract}
The prevention and control of pain are basic components to have an adequate anesthesia. The aim of this study was to investigate the postoperative analgesia of ketamine in subanestesica dose, of methadone and the association of both intramuscularly in cats. A total of 24 cats proven healthy were referred for elective ovariossalpingohisterectomia. The day before the beginning of the experiment animals were anesthetized for placing a catheter in the jugular vein for subsequent blood sampling for measurement of serum concentration of cortisol. The next day, animals were randomly allocated to three groups $(n=8)$, which received ketamine $(G C)$ at a dose of $0.5 \mathrm{mg} / \mathrm{kg}$; methadone $(\mathrm{GM})$ at a dose of $0.3 \mathrm{mg} / \mathrm{kg}$; methadone and ketamine (GCM) at doses of $0.5 \mathrm{mg} / \mathrm{kg}$ and $0.3 \mathrm{mg} / \mathrm{kg}$, respectively, all in the intramuscular route. All groups received the drug 20 minutes before induction of anesthesia, which was performed with propofol and maintained with isoflurane. The parameters evaluated were the measurement of cortisol in moments MO (baseline-before surgery), M1(intraoperative), M2, M3, M4, M8, M12 and M24 (2,3,4,8,12 and 24 hours after the end of surgery) and evaluation of pain score by multidimensional scale of acute pain in
\end{abstract}

Recebido em 9 de junho de 2013

Aceito em 19 de fevereiro de 2014

E-mail: vanessa.sasso@hotmail.com 
cats in the moments M0, M2, M3, M4, M8, M12 and M24. The cortisol levels were higher in GC compared to GM and GCM in M2 and M3. Pain scores were higher in GC in moments M2 and M3 compared to GM and GCM, respectively. In summation of points in M2, GC showed higher values than GM and GCM, and M4, GC obtained values greater than GCM. Comparing the number of rescues postoperatively in GC 8/8 of animals required rescue, in GM /8 and in GCM 3/8. It was concluded that the combination of methadone and ketamine in cats undergoing elective OSH provides adequate postoperative analgesia, reducing the requirement for analgesics in the postoperative period.

Keywords: cat, ketamine, methadone, analgesia

\section{INTRODUÇÃO}

O manejo da dor é mais facilmente realizado nos casos de trauma ou cirurgias, ou seja, quando a dor possui caráter agudo (Hellyer et al., 2007). Nesse contexto, a analgesia preventiva tem se destacado por reduzir ou prevenir a dor pósoperatória e por modular a hipersensibilidade dos neurônios do corno dorsal da medula espinhal decorrente de estímulos nociceptivos prolongados, por meio da administração de analgésicos (Otero, 2005). Embora o tratamento da dor seja um tópico emergente e popular na medicina veterinária, o uso de analgésicos em gatos tem recebido pouca atenção em comparação aos cães (Hellebrekers, 2002). Algumas das dificuldades residem na avaliação e na percepção da dor na espécie felina. A simples observação do gato muitas vezes não gera resultados acurados, pois a estrutura social dos felinos não facilita a expressão da dor (Hellebrekers, 2002). Nesse sentido, a escala multidimensional de dor aguda em felinos (Brondani et al., 2011) tem se mostrado mais sensível e específica para utilização em gatos.

A metadona, além da sua ação agonista em receptores do tipo $\mu$, atua como antagonista dos receptores do tipo NMDA, sendo útil nos casos de hiperalgesia e tolerância a opioides (Wagner, 2009). A dose de cetamina necessária para produzir efeito analgésico é consideravelmente menor do que aquela necessária para produzir anestesia, o que explica por que esse anestésico conserva propriedades anti-hiperalgésicas mesmo em doses subanestésicas (Harper, 2007).

Dessa forma, o objetivo do presente estudo foi avaliar o efeito analgésico da cetamina em doses subanestésicas, da metadona, ou da associação de ambas em gatas submetidas à ovariossalpingohisterectomia eletiva, utilizando-se uma escala multidimensional de dor aguda e da dosagem de cortisol sérico.

\section{MATERIAL E MÉTODOS}

Este estudo foi aprovado pelo Comitê de Ética e de Bem-Estar Animal da instituição de origem

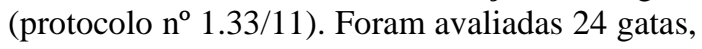
adultas, mestiças, com peso médio de $2,8 \pm 0,6 \mathrm{~kg}$, encaminhadas para OSH eletiva. Os proprietários dos animais assinaram um termo de consentimento para participação desses animais na pesquisa. Foram realizados exames físicos e laboratoriais (hemograma, ureia, creatinina, fosfatase alcalina, alanina aminotransferase, gama-glutamiltransferase e albumina) para constatação de higidez do paciente.

Previamente ao início do estudo, os animais foram alojados em uma sala silenciosa, com área de $8 \mathrm{~m}^{2}$, contendo uma gaiola de metal $\left(0,8 \mathrm{~m}^{3}\right)$ e com espaço para interação do observador com o animal por um período mínimo de dois dias para adaptação ao local e conhecimento dos seus hábitos, os quais foram avaliados de acordo com a escala multidimensional de dor aguda em felinos. A alimentação foi à base de ração comercial e água ad libitum.

A instrumentação foi realizada 24 horas antes do início do estudo; para tal, realizou-se jejum sólido de 12 horas e hídrico de seis horas. Nessa fase, a indução anestésica foi realizada com propofol (Propovan, Cristália, Itapira/SP Brasil) dose efeito $(7,8 \pm 1,6 \mathrm{mg} / \mathrm{kg})$. Em seguida, os animais foram intubados com sonda tipo Murphy adequada ao seu porte, conectados ao sistema sem reinalação de gases (circuito de Baraka) e mantidos em anestesia inalatória com isoflurano (Isoforine, Cristália, Itapira, SP, Brasil) diluído em $100 \%$ de oxigênio com um fluxo de oxigênio de $200 \mathrm{~mL} / \mathrm{kg} / \mathrm{min}$. A concentração de isoflurano foi avaliada por meio de um analisador de gases (Monitor de gases anestésicos Poet IQ2 ${ }^{\circledR}$ Criticare Systems, Inc, Waukesha, USA), sendo sua concentração mantida de acordo com o plano 
anestésico do paciente, o qual foi verificado por meio de reflexo palpebral e tônus mandibular. Em seguida, foi realizada tricotomia da veia jugular e da região abdominal.

O animal foi posicionado em decúbito dorsal para colocação do cateter Intracath 19G (Cateter Intravenoso central, Becton e Dickinson Giromed - Campinas - SP, Brasil) na veia jugular direita, o qual foi utilizado para as coletas de sangue para posterior análise do cortisol. O cateter foi fixado por meio de pontos de pele, e o animal permanecia com uma faixa elástica $(3 \mathrm{M}$ Vetrap ${ }^{\circledR}$, Bandaging tape, 3M Animal Care products, St.Paul, Minnesota, USA) para sua proteção. Decorridas 24 horas, os animais foram alocados aleatoriamente em três grupos: grupo GC $(\mathrm{n}=08)$ recebeu $0,5 \mathrm{mg} / \mathrm{kg}$ de cetamina (Vetaset®, Fort Dodge Saúde Animal, Campinas, SP, Brasil); GM (n=08) recebeu metadona (Mytedom ${ }^{\circledR}$, Cristália, Itapira, SP, Brasil) na dose de $0,3 \mathrm{mg} / \mathrm{kg}$; e GCM $(\mathrm{n}=08)$ recebeu $0,5 \mathrm{mg} / \mathrm{kg}$ e $0,3 \mathrm{mg} / \mathrm{kg}$ de cetamina e metadona, respectivamente. Todos os grupos receberam os fármacos 20 minutos antes da indução anestésica, pela via intramuscular.

Decorridos os 20 minutos, realizava-se a indução anestésica com propofol dose efeito pela via intravenosa (IV) $(7,7 \pm 0,5 \mathrm{mg} / \mathrm{kg})$ e iniciava-se a administração de solução de cloreto de sódio na taxa de $10 \mathrm{~mL} / \mathrm{kg} / \mathrm{h}$. Após a intubação orotraqueal, os animais eram conectados ao sistema sem reinalação de gases (circuito de Baraka) e mantidos em anestesia geral inalatória com isoflurano diluído em $100 \%$ de oxigênio com fluxo de $200 \mathrm{~mL} / \mathrm{kg} / \mathrm{min}$. A concentração expirada de isoflurano foi avaliada por meio de um analisador de gases (Monitor de gases anestésicos Poet IQ2 ${ }^{\circledR}$ Criticare Systems, Inc, Waukesha, USA), sendo sua concentração mantida de acordo com o plano anestésico do paciente, o qual foi verificado por meio de reflexo palpebral e tônus mandibular. A monitoração anestésica foi realizada com auxílio de monitor multiparamétrico (Dixtal DX210 ${ }^{\circledR}$ Brasil Indústria e Comércio Ltda., Manaus, AM, Brasil), em que se avaliaram frequência cardíaca (FC), frequência respiratória $(f)$, pressão parcial de dióxido de carbono ao final da expiração $\left(\mathrm{EtCO}_{2}\right)$ e saturação de oxi-hemoglobina $\left(\mathrm{SpO}_{2}\right)$, além da pressão arterial sistólica (PAS) por meio de Doppler vascular portátil (Modelo 841-A, Parks Medical Electronics ${ }^{\circledR}$, Inc. Aloha, Oregon,
USA), com a utilização de manguitos neonatais de tamanhos um ou dois. Os parâmetros acima citados foram avaliados no período transcirúrgico, nos seguintes momentos: T0 (imediatamente antes do início da cirurgia), T1, T2 e T3 (cinco, 10 e 15 minutos após o início do procedimento, respectivamente). $\mathrm{O}$ resgate analgésico transoperatório foi realizado com fentanil pela via intravenosa (Fentanest@, Cristália, Itapira, SP, Brasil), na dose de $5 \mathrm{ug} / \mathrm{kg}$, quando ocorresse aumento da pressão arterial sistólica e/ou da frequência cardíaca e/ou respiratória acima de $20 \%$ comparado ao basal (M0). O procedimento cirúrgico foi realizado sempre pelo mesmo cirurgião, o qual utilizou a técnica das três pinças (Hedlund, 2002), e foi padronizado em 20 minutos.

A avaliação pós-operatória constou da mensuração do cortisol e do escore de dor por meio da escala multidimensional de dor aguda em felinos (Brondani et al., 2011). A soma total de pontos da escala é 30; quando o resultado de um dos avaliadores fosse igual ou maior que oito, era realizado resgate analgésico com morfina (Dimorf®, Cristália, Itapira, SP, Brasil), na dose de $0,2 \mathrm{mg} / \mathrm{kg}$, pela via intramuscular.

O animal foi avaliado de acordo com a escala uma hora antes do procedimento cirúrgico; e no período pós-operatório com duas (M2), três (M3), quatro (M4), oito (M8), 12 (M12) e 24 (M24) horas do término do procedimento. Todas as avaliações foram realizadas pelos mesmos dois avaliadores (A e B), os quais eram cegos ao tratamento, e o resgate analgésico pós-operatório era realizado mesmo quando apenas um dos avaliadores tinha atingido os pontos necessários para efetuá-lo. Os animais eram avaliados nos momentos seguintes, ainda que tivessem recebido resgate analgésico.

A amostra para mensuração do cortisol foi coletada uma hora antes da cirurgia (M0), no transcirúrgico, após o pinçamento do primeiro pedículo (M1), e no pós-operatório, com duas (M2), três (M3), quatro (M4), oito (M8), 12 (M12) e 24 (M24) horas após o final do procedimento. Coletou-se $1,5 \mathrm{~mL}$ de sangue venoso do cateter Intracath, o qual foi colocado em tubo seco com ativador de coágulo e centrifugado. $\mathrm{O}$ soro foi armazenado e congelado a $-80^{\circ} \mathrm{C}$ em tubos Eppendorf na forma de triplicata. A análise das amostras foi realizada 
em laboratório comercial, sendo utilizado o método de radioimunoensaio, com detecção mínima de 0,06ug/dL.

Os dados paramétricos foram submetidos à análise de variância de uma via (One Way ANOVA), seguida pelo teste Student-NewmanKeuls para comparação entre grupos. Para comparação entre tempos dentro do mesmo grupo, utilizou-se a análise de variância de uma via com repetições múltiplas (One Way ANOVA), seguida pelo teste Student-NewmanKeuls. Para os dados não paramétricos, utilizouse o teste de Friedman, seguido pelo teste de Tukey para comparação entre os tempos dentro do mesmo grupo, e o teste de Kruskal-Wallis, seguido pelo teste de Tukey para comparação entre os grupos. As diferenças foram consideradas significativas quando $\mathrm{P}<0,05$.

\section{RESULTADOS E DISCUSSÃO}

O peso médio dos animais em GC, GM e GCM foi de $3,1 \pm 0,6 \mathrm{~kg}, \quad 2,8 \pm 0,8 \mathrm{~kg}$ e $2,7 \pm 0,4 \mathrm{~kg}$, respectivamente, não havendo diferença significativa entre grupos. $\mathrm{O}$ procedimento cirúrgico teve duração média de $18,4 \pm 4,0$ minutos.

Não foram observadas diferenças significativas entre os grupos para os valores basais de hemograma e bioquímicos, para o número de aplicações de fentanil e para os valores de FC, $f$, $\mathrm{PAS}, \quad \mathrm{SpO}_{2}, \quad \mathrm{EtCO}_{2}$ e EtISO no período transoperatório. Como efeitos adversos da medicação pré-operatória administrada, observou-se excitação apenas em um animal, no pré-operatório, após a aplicação de cetamina e metadona intramuscular, mas não foi necessário excluir o animal do estudo.

A administração de opioides em gatos acordados, geralmente, é realizada em doses baixas e é restrita à medicação pré ou pós-anestésica devido ao receio de causar reações de excitação quando esses fármacos são usados em doses altas (Taylor; Robertson, 2004). Na forma racêmica, a metadona tem sido recomendada, em doses que variaram de 0,1 a $0,6 \mathrm{mg} / \mathrm{kg}$ (Bley et al., 2004). Bley et al. (2004) observaram excitação durante a recuperação pós-anestésica de alguns gatos que receberam $0,6 \mathrm{mg} / \mathrm{kg}$ de metadona pela via intramuscular. Bernardi et al. (2012), em seu estudo, relatam apenas um caso de euforia durante a recuperação pós-anestésica com o uso de $0,2 \mathrm{mg} / \mathrm{kg}$ de metadona pela via intramuscular. De acordo com Ferreira (2010), 6/8 gatos que receberam $0,3 \mathrm{mg} / \mathrm{kg}$ de metadona pela via intravenosa apresentaram sedação entre um e cinco minutos, em seguida estes apresentaram um período eufórico de duração variável, compreendido entre 20 minutos e seis horas após a administração da metadona.

Já a dose de cetamina é considerada subanestésica quando menor que $2 \mathrm{mg} / \mathrm{kg} \mathrm{em}$ bolus pela via intramuscular e menor que $1 \mathrm{mg} / \mathrm{kg}$ em bolus pela via intravenosa ou peridural (Suzuki et al., 1999). Devido ao fato de a cetamina bloquear de maneira não competitiva os receptores NMDA, e considerando que quanto menor sua dose, maior sua afinidade por esses receptores (Suzuki et al., 1999), o presente estudo preconizou a utilização de $0,5 \mathrm{mg} / \mathrm{kg}$ pela via intramuscular, o que caracteriza uma dose subanestésica.

No pós-operatório, o GC apresentou valores maiores de cortisol em relação ao GM e ao GCM nos momentos M2 e M3, mas não houve diferença significativa entre GM e GCM (Fig. 1). Entre os momentos dentro do grupo, observou-se aumento dos valores de cortisol no GC no momento M2 em relação ao M0. No GM e no GCM, não houve diferença significativa entre os momentos.

O tratamento da dor em gatos tem sido historicamente negligenciado, no entanto existem evidências de que esse cenário vem mudando (Williams et al., 2005). Uma das primeiras causas para esse fato é a dificuldade em identificar e quantificar a intensidade da dor em gatos (Brondani et al., 2011), em razão de sinais inexpressivos de desconforto, bem como da ausência de outras alterações fisiológicas. No presente estudo, a metodologia utilizada incluiu a escala multidimensional de dor aguda em felinos e a avaliação de resposta neuroendócrina por meio da mensuração de cortisol, pois, de acordo com Cambridge et al. (2000), a mensuração de variáveis neuroendócrinas, como betaendorfina, catecolaminas e concentração de cortisol, tem sido correlacionada com a dor em gatos. No entanto, outros fatores além da dor, como alterações no ambiente de internação e fisiológicas resultantes da anestesia e da cirurgia, 
podem afetar a resposta neuroendócrina (Lin et al., 1993).

A avaliação do animal antes do estímulo cirúrgico é de extrema importância devido aos diferentes comportamentos dos gatos. A metodologia proposta neste estudo enfatiza a avaliação basal do paciente, sendo que todos passaram por avaliação por meio da escala de dor e da coleta de sangue para mensuração de cortisol antes do estímulo cirúrgico. Os níveis de cortisol basal mantiveram-se dentro dos limites fisiológicos para a espécie felina, que estão

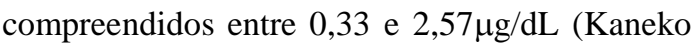
et al., 1997), o que demonstra que as coletas foram realizadas sem provocar estresse nos animais, pois as médias da concentração de cortisol foram de 1,$71 ; 1,00$ e 1,06 para os grupos GC, GM e GCM, respectivamente. De acordo com Steagall et al. (2007), o parâmetro neuroendócrino isolado é inadequado para a avaliação da dor, embora possa contribuir para o quadro geral. Para tanto, outro dado que mostra a tranquilidade dos animais com os avaliadores é a aferição da pressão arterial sistólica, que no basal já apresentou valores baixos, sendo 128, 132 e $129 \mathrm{mmHg}$ as médias nos grupos GC, GM e GCM, respectivamente.

A coleta de sangue para dosagem de cortisol transcirúrgico (M1) foi sempre realizada após o pinçamento do primeiro pedículo ovariano, momento que exigiu a administração de fentanil em todos os animais de todos os grupos, por isso, nesse momento, a concentração sérica de cortisol não apresentou diferença significativa entre os grupos. De acordo com Robertson et al. (2005a), a concentração de fentanil no plasma de gatos se correlacionou bem com a analgesia, pois, após dois minutos da administração de $10 \mu \mathrm{g} / \mathrm{kg}$ pela via intravenosa, a concentração plasmática de fentanil atingiu níveis máximos, o que corrobora os resultados encontrados neste estudo, em que, após a administração de fentanil, os valores de cortisol mantiveram-se dentro dos limites fisiológicos.

A recuperação da cirurgia foi realizada em sala adaptada e própria para gatos, onde o animal permanecia na caixa de transporte, em ambiente escuro e sem estímulos auditivos. Os animais eram colocados na gaiola somente após mostrarem-se tranquilos, evitando a ocorrência de qualquer tipo de ferimento. Inicialmente os escores de dor no momento uma hora pósoperatória eram avaliados, mas, devido à sedação e à impossibilidade de avaliação dos animais nesse período, esse momento foi descartado da estatística.

Os escores de dor de acordo com o cortisol e o somatório total de pontos (Fig. 2) foram maiores no GC em relação aos grupos GM e GCM nos momentos M2 e M3. A partir desses momentos, entretanto, houve declínio do cortisol e da pontuação devido ao resgate aplicado quando a pontuação na escala era igual ou maior que oito. $\mathrm{O}$ número de resgates com morfina foi maior no GC, sofrendo redução gradativa no GM e no GCM. Com 12 e 24 horas pós-operatórias, a maioria dos animais não apresentava mais nenhum sinal de dor.

A aplicação do resgate analgésico no pósoperatório (Tab. 1) teve relação com a diminuição da pontuação total da escala e do cortisol do momento posterior, pois, após a aplicação da morfina, o animal era avaliado no próximo momento da mesma forma. Quando a avaliação era feita de hora em hora, aguardava-se pelo menos 30 minutos para a próxima avaliação, para que a morfina já pudesse ter efeito analgésico.

A metadona apresenta ação agonista em receptores opioides, afinidade pelos receptores NMDA e possui ação inibitória na recaptação da norepinefrina e da serotonina (Gorman et al., 1997). A combinação sinérgica nos receptores opioides com o antagonismo não competitivo em NMDA parece promover o bloqueio efetivo da sensibilização central. Além disso, a ação antagonista NMDA parece estar associada ao bloqueio da hiperalgesia imediata e tardia (Holtman e Wala, 2007). A cetamina age sobre uma variedade de receptores, entre eles se destaca o antagonismo não competitivo dos receptores NMDA (Suzuki et al., 1999), da mesma forma que a metadona. A utilização de associações de analgésicos é chamada de tratamento analgésico multimodal, sendo mais eficaz que o uso de drogas isoladas no controle da dor aguda após OSH em gatas (Pogatzki-Zahn et al., 2006). 


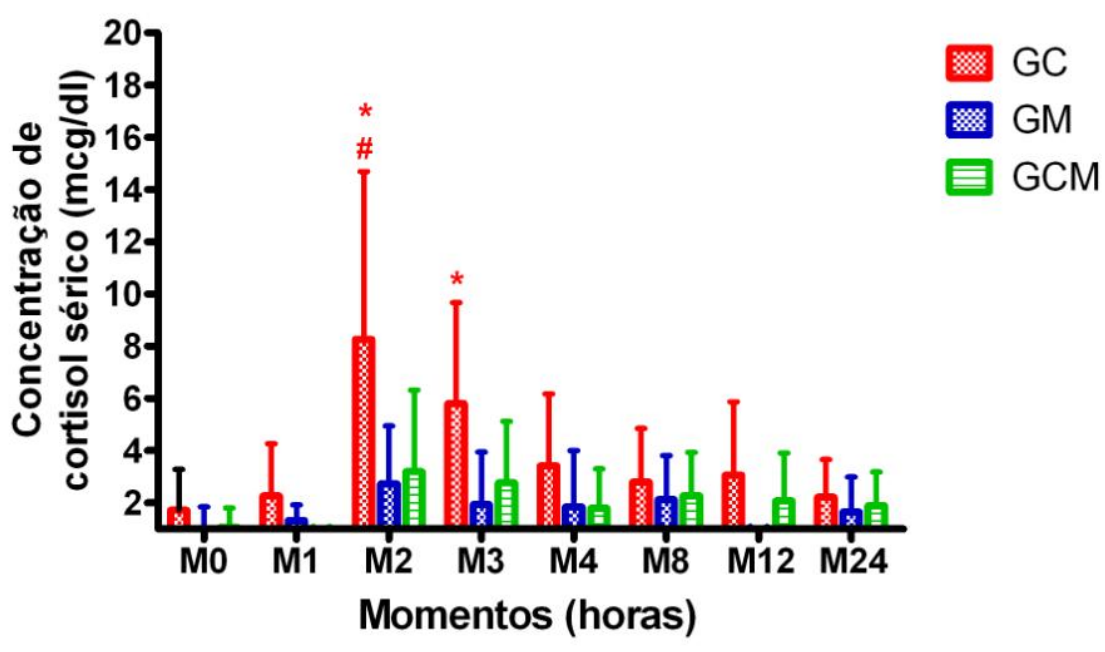

M0 - basal; M1 - transcirúrgico; M2, M3, M4, M8, M12 e M24 - duas, três, quatro, oito, 12 e 24 horas após o término do procedimento cirúrgico.

Figura 01. Valores médios e desvios-padrão da concentração sérica de cortisol ( $\mu \mathrm{g} / \mathrm{dL})$ de gatas submetidas a OSH, tratadas com cetamina (GC - 0,5mg/kg, $\mathrm{n}=08)$; metadona (GM - 0,3mg/kg, $\mathrm{n}=08)$; ou cetamina e metadona (GCM - 0,5mg/kg e $0,3 \mathrm{mg} / \mathrm{kg}$, respectivamente, $\mathrm{n}=08)$, pela via intramuscular. \# Difere de M0, ANOVA uma via, seguido pelo teste SNK $(\mathrm{P} \leq 0,05)$. *Difere dos demais grupos, ANOVA uma via $\mathrm{RM}$, seguido pelo teste $\mathrm{SNK}(\mathrm{P} \leq 0,05)$.

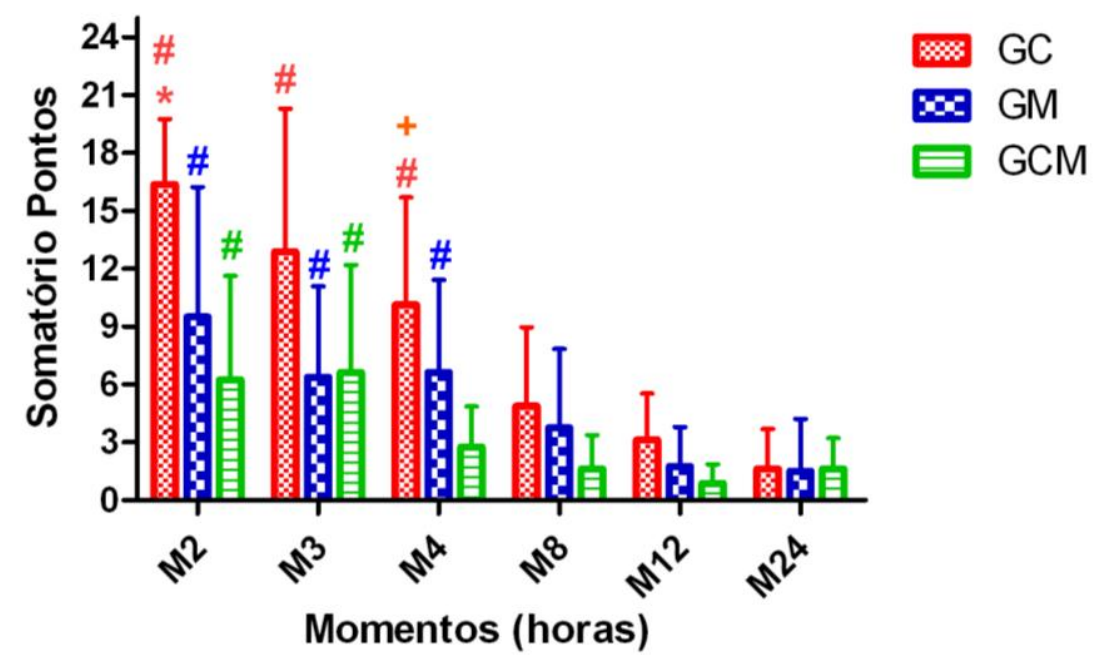

M0 - basal; M1 - transcirúrgico; M2, M3, M4, M8, M12 e M24 - duas, três, quatro, oito, 12 e 24 horas após o término do procedimento cirúrgico.

Figura 2. Valores médios e desvios-padrão do somatório de pontos da escala multidimensional de dor aguda, de gatas submetidas a OSH, tratadas com cetamina (GC - 0,5mg/kg); metadona (GM - 0,3mg/kg); ou cetamina e metadona (GCM - 0,5mg/kg e $0,3 \mathrm{mg} / \mathrm{kg}$, respectivamente), pela via intramuscular. ${ }^{\#}$ Difere de M0, ANOVA teste de Friedman, seguido pelo teste de Tukey $(\mathrm{P} \leq 0,05)$. *Difere de todos os grupos. ${ }^{+}$ Difere apenas de GCM, teste de Kruskal-Wallis, seguido pelo teste de Tukey $(\mathrm{P} \leq 0,05)$. 
Tabela 1. Percentual dos animais que receberam resgate pós-operatório com morfina, submetidos a ovariossalpingo-histerectomia, tratados com cetamina (GC - 0,5mg/kg); metadona (GM - 0,3mg/kg); ou cetamina e metadona (GCM - 0,5mg/kg e $0,3 \mathrm{mg} / \mathrm{kg}$, respectivamente), pela via intramuscular

\begin{tabular}{lcccccc}
\hline Grupos & \multicolumn{7}{c}{$\%$ Animais } \\
Tempo (h) & $2(\mathrm{M} 3)$ & $3(\mathrm{M} 4)$ & $4(\mathrm{M} 5)$ & $8(\mathrm{M} 6)$ & $12(\mathrm{M} 7)$ & $24(\mathrm{M} 8)$ \\
GC & $100(8 / 8)$ & $75(6 / 8)$ & $62,5(5 / 8)$ & $37,5(3 / 8)$ & $0(0 / 8)$ & $0(0 / 8)$ \\
GM & $50(4 / 8)$ & $50(4 / 8)$ & $25(2 / 8)$ & $12,5(1 / 8)$ & $0(0 / 8)$ & $0(0 / 8)$ \\
GCM & $25(2 / 8)$ & $25(2 / 8)$ & $12,5(1 / 8)$ & $0(0 / 8)$ & $0(0 / 8)$ & $0(0 / 8)$ \\
\hline
\end{tabular}

De acordo com as diretrizes para uso da escala, inicialmente o animal era avaliado dentro da gaiola; posteriormente, ela era aberta para se observar o comportamento no ambiente e com o avaliador. O animal, então, era sempre encorajado a movimentar-se para se avaliar a atividade ou a relutância em mover-se. Ainda, os sinais de dor em um felino incluem postura curvada, cabeça baixa, ausência de resposta a estímulos externos, mordida ou lambida excessiva da área dolorosa, redução do apetite e dos hábitos de higiene, alteração da postura de micção e às vezes vocalização (Robertson, 2005b). Além das variáveis comportamentais, alterações fisiológicas, como a PAS, também foram avaliadas, porém Mathews (2000) relata que parâmetros fisiológicos nem sempre são fidedignos, pois qualquer situação que leve ao estresse, ou até mesmo a utilização de fármacos antes, durante ou após os procedimentos cirúrgicos, pode desencadear instabilidade nessas variáveis.

Duas horas após a cirurgia (M2), os valores de cortisol e a pontuação na escala foram maiores nos grupos GC em relação ao GM e ao GCM. Nesse momento, $100 \%$ dos animais do GC receberam resgate, $50 \%$ do GM e $25 \%$ do GCM (Tab. 1). A PAS e a vocalização à dor não apresentaram diferença significativa entre os grupos e entre os momentos no pós-operatório. A PAS pode não ter sofrido alteração devido à influência do resgate com a morfina que foi aplicada no momento anterior. A dor pode ter efeitos sobre o sistema cardiovascular, resultando em vasoconstrição, elevação na pressão sanguínea, aumento da contratilidade e da frequência cardíaca e maior susceptibilidade a arritmias cardíacas (Cousins, 1994). Entretanto, devido à utilização de morfina como resgate analgésico, esta pode ter mascarado o aumento da pressão sanguínea, pois, de acordo com Lamont e Mathews (2007), a maioria dos opioides reduz a frequência cardíaca, o débito cardíaco e a pressão arterial quando esses fármacos são utilizados em doses analgésicas. A morfina tem sido amplamente usada em gatos nas doses de 0,1 a $0,2 \mathrm{mg} / \mathrm{kg}$, sendo eficaz na analgesia sem promover excitação (Lascelles e Waterman, 1997), o que corrobora a dose utilizada neste estudo como resgate analgésico.

A avaliação em três horas (M3) pós-operatória foi similar à de duas horas (M2), com os valores de cortisol maiores no GC em comparação ao GM e ao GCM. Os animais que receberam resgate no momento anterior obtiveram pontuação menor e valores de cortisol também menores, porém alguns ainda necessitaram de resgate.

As avaliações seguintes (quatro até 24 horas) não apresentaram diferença significativa em nenhuma variável, no entanto alguns animais ainda receberam resgate. Com 12 e 24 horas de avaliação nenhum dos animais recebeu resgate; ou seja, até oito horas pós-operatória, os animais necessitavam de analgesia complementar, pois, nas próximas avaliações, que foram após quatro e 16 horas depois do último resgate, não havia mais efeito da morfina ou apenas efeito residual. Segundo Taylor et al. (2001), em felinos, a meiavida plasmática da morfina pela via intramuscular é de 93,6 minutos, com intervalo posológico de aproximadamente quatro a seis horas. Esse período é prolongado devido à deficiência de glicuroniltransferase nessa espécie (Otero, 2005).

Dois animais do GC apresentaram euforia/ disforia, um com quatro horas e outro com oito horas pós-operatória, devido aos resgates consecutivos com morfina. No momento da avaliação, esses animais já haviam recebido 0,6 e $0,4 \mathrm{mg} / \mathrm{kg}$ de morfina, respectivamente. Outros animais do mesmo grupo chegaram a receber a 
$1,0 \mathrm{mg} / \mathrm{kg}$ de morfina, porém não apresentaram sinais de euforia/disforia. Segundo Robertson (2008), mesmo com a dosagem adequada, os efeitos comportamentais geralmente incluem euforia, como ronronar e amassar com as patas dianteiras. Deve-se considerar a variação individual de dor entre os animais, o que pode ter levado a alguns animais receberem doses mais altas de morfina e não apresentarem excitação.

No presente estudo, houve redução no consumo de opioides no pós-operatório quando a associação da cetamina e metadona foi utilizada; no total, o GCM recebeu $1,4 \mathrm{mg}$ de morfina, o GM 2,2mg e o GC 5,6mg. Os resultados deste estudo corroboram os achados de Pacreu et al. (2012), em que a administração de cetamina associada à metadona em humanos submetidos a artrodese lombar não melhorou a analgesia pósoperatória imediata, porém, durante as 48 horas de avaliação, os pacientes que receberam a combinação dos fármacos necessitaram de $70 \%$ a menos de opioide quando comparados àqueles do grupo que recebeu metadona isolada.

Apesar de o GC somar mais pontos na escala e apresentar valores mais altos de cortisol quando comparado ao GM e ao GCM, ainda se pode sugerir efeito analgésico desse fármaco, pois a maior pontuação atingida na escala foi de 22 pontos, ou seja, nenhum animal apresentou a maior dor possível segundo a escala (30 pontos). Entretanto essa hipótese só poderia ser afirmada caso houvesse um grupo placebo, isto é, um grupo que não recebesse nenhum tipo de fármaco analgésico.

Apesar de o GCM apresentar o menor número de resgate e somatório de pontos, os valores de cortisol foram iguais ao de GM. No GCM, três animais necessitaram de resgate, totalizando sete aplicações, e um animal apresentava excitação no momento em que recebeu resgate. Já no GM, cinco animais necessitaram de resgate, totalizando 11 aplicações. Os valores de cortisol podem ter sofrido influência de diversos fatores. Um deles diz respeito à pequena amostra de animais utilizada no estudo. Além disso, fatores ambientais podem interferir nos níveis de cortisol, visto que a escala utilizada é de grande sensibilidade para a dor aguda em felinos.

As principais limitações do presente estudo foram o número de animais. Dessa forma, para estudos futuros, sugere-se maior número de animais para que a variabilidade interindividual seja desconsiderada. Além disso, a avaliação da dor pós-operatória na primeira hora após o procedimento é difícil em virtude de os animais ainda estarem se recuperando da anestesia, o que pode confundir o avaliador. Assim, sugere-se avaliação da dor utilizando-se a escala multidimensional de dor aguda apenas a partir da segunda hora após o procedimento.

\section{CONCLUSÕES}

Conclui-se que a associação de metadona e cetamina em gatas submetidas à OSH eletiva promove analgesia pós-operatória adequada e, portanto, reduz o requerimento de analgésicos no período pós-operatório. A administração de cetamina e metadona isoladas não produz analgesia pós-operatória satisfatória em gatas submetidas a OSH.

\section{REFERÊNCIAS}

BERNARDI, C.A.; CASSU, R.N.; BALAN, J.A.O. et al. Analgesia pós-operatória com metadona em gatos: administração epidural e intramuscular. Arq. Bras. Med. Vet. Zootec., v.64, p.45-52, 2012.

BLEY, C.R.; NEIGER-AESCHBACHER, G.; BUSATO, A. et al. Comparison of perioperative racemic methadone, levo-methadone and dextromoramide in cats using indicators of postoperative pain. Vet. Anaest. and Anal., v.31, p.175182, 2004.

BRONDANI, J.T.; LUNA, S.P.L.; PADOVANI, C.R. Refinement and initial validation of a multimensional composite scale for use in assessing acute postoperative pain in cats. Am. J. Vet. Res., v.72, p.174-183, 2011.

CAMBRIDGE, A.J.; TOBIAS, K.M.; NEWBERRY, R.C. et al. Subjective and objective measurements of postoperative pain in cats. J. Am. Vet. Medical Associat., v.217, p.685-690, 2000.

COUSINS, M. Acute and postoperative pain. IN: Wall, P.D.; Melzack, R. Textbook of pain. Edinburgh: Churchill Livingstone, 1994. p.357-385.

FERREIRA, T.H. A Farmacocinética da metadona e seus efeitos antinociceptivos, comportamentais e sobre a concentração alveolar minima de sevofluorano em felinos. 2010. 122f. Tese (doutorado em anestesiologia). Universidade Estadual Paulista "Júlio de Mesquita Filho”, Campus de Botucatu, SP. 
GORMAN, A.L.; ELLIOTT, K.J.; INTURRISI, C.E. The $\mathrm{d}$ - and $\mathrm{l}$ - isomers of methadone bind to the noncompetitive site on the $\mathrm{N}$-methyl-d-aspartate (NMDA) receptor in rat forebrain and spinal cord. Neurosci. Lett., v.223, p.5-8, 1997.

HARPER, T.D. Perioperative uses of low-dose ketamine for pain management. Plastic Surgical Nursing, v.27, p.98-101, 2007.

HEDLUND, S.C. Cirurgia dos sistemas reprodutivo e genital. IN: FOSSUM, W.T. (Ed). Cirurgia de pequenos animais. São Paulo: Roca, 2002. p.571-637.

HELLEBREKERS, L.H. Dor em animais. Manole: Sao Paulo, 2002. p.69-79.

HELLYER, P.W.; ROBERTSON, S.A.; FAILS, A.D. Pain and its management. IN: TRANQUILLI W.J.; THURMON J.C.; GRIMM K.A., editors LUMB \& JONES Veterinary Anaesthesia and Analgesia. 4. ed. Blackwell Publishing, cap.3, 2007. p.31-53.

HOLTMAN, J.R.; WALA, E.L. Characterization of the antinociceptive and pronociceptive effects of methadone in rats. Anesthesiology, v.106, p.563-571, 2007.

KANEKO, J.J.; HARVEY, J.W.; BRUSS, M.L. Clinical Biochemistry of Domestic Animals. San Diego: Academic Press, 1997. p.896.

LAMONT, L.A.; MATHEWS, K. Opioids, nonsteroidal anti-inflamatories, and analgesic adjuvants. IN: TRANQUILLI, W.J.; THURMON, J.C.; GRIMM, K.A. (Eds): Lumb and Jones 'Veterinary Anesthesia and Analgesia. 4.ed., Ames: Blackwell Publishing, 2007. p.241-271.

LASCELLES, D.; WATERMAN, A. Analgesia in cats. IN Pract., v.19, p.203-213, 1997.

LIN, H.C.; BENSON, G.J.; THURMON, J.C. et al. Influence of anesthetic regimens on the postoperative catecholamine response associated with onychectomy in cats. Am. J. Vet. Res., v.54, p.1721-1724.1993.

MATHEWS, K.A. Pain assessment and general approach to management. Vet. Clinics of North Am. Small Anim. Pract., v.30, p.729-755, 2000

OTERO, P.E. Dor avaliação e tratamento em pequenos animais. Intermédica Editorial: São Caetano do Sul, SP, 2005. p.76-108, 132-140, 158-159, 202204.
PACREU, S.; CANDIL, J.F.; MOLTÓ, L. et al. The perioperative combination of methadone and ketamine reduces post-operative opioid usage compared with methadone alone. Acta Anaesthesiol. Scandinav., v.56, p.1250-1256, 2012.

POGATZKI-ZAHN, E.M.; ZAHN, P.K. From preemptive to preventive analgesia. Curr. Opin Anaesthesiol., v.19, p.551-555, 2006.

ROBERTSON, S.A. Assessment and management of acute pain in cats. J. Vet. Emerg. Crit. Care., v.15, p.261-272, 2005b

ROBERTSON, S.A. Managing Pain in Feline Patients. Vet. Clin. Small Anim., v.38, p.1267-1290, 2008.

ROBERTSON, A.; TAYLOR, P.M.; SEAR, L.W. et al. Relationship between plasma concentrations and analgesia after intravenous fentanyl and disposition after other routes of administration in cats. J. Vet. Pharmacol. Ther., v.28, p.87-93, 2005a.

STEAGALL, P.; TAYLOR, P.M.; BRONDANI, J.T. et al. Effects of buprenorphine, carprofen and saline on thermal and mechanical nociceptive thresholds in cats. Vet. Anaest. and Analg. v.34, p.344-350. 2007.

SUZUKI, M.; TSUEDA, K.; LANSING, P.S. et al Small-dose ketamine enhances Morphine-induced analgesia after outpatient surgery. Anesth. Analg., v.89, p.98-103. 1999.

TAYLOR, P.M.; ROBERTSON, S.A. Pain management in cats - past, present and future. Part 1. The cat is unique. J. Fel. Med. Surg, v.6, p.313-320. 2004.

TAYLOR, P.M.; ROBERTSON, S.A.; DIXON, M.J. et al. Morphine, pethidine and buprenorphine disposition in cat. J. Vet. Pharm. and Therap., v.24, p.391 - 398, 2001.

WAGNER, A.E. Opioides. IN: Gaynor, J.S.; Muir III, W.W. Manual de Controle da dor em Medicina Veterinária, 2.ed. São Paulo: Med Vet, cap. 9, 2009. p.163-182.

WILLIAMS, V.M.; LASCELLES, B.D.X.; ROBSON, M.C. Current attitudes to, and use of, peri-operative analgesia in dogs and cats by veterinarians in New Zealand. New Z. Vet. J., v.53, p.193-202. 2005. 